\title{
A new method for yeast phagocytosis analysis by flow cytometry
}

\author{
Catarina Carneiro, Catarina Vaz, Joana Carvalho-Pereira, Célia Pais, Paula Sampaio* \\ Centre of Molecular and Environmental Biology (CBMA), Department of Biology, University of Minho, Braga, Portugal
}

\section{A R T I C L E I N F O}

\section{Article history:}

Received 29 January 2014

Received in revised form 25 March 2014

Accepted 25 March 2014

Available online 3 April 2014

\section{Keywords:}

Candida spp

Flow cytometry

Sytox Green

Propidium iodide

Phagocytosis

PAMPS

\begin{abstract}
A B S T R A C T
Herein we developed a method based on the quenching effect of propidium iodide over Sytox-Green fluorescence to assess yeast phagocytosis by flow cytometry. It allows accurate quantification of living from dead phagocytes; internalized from non-internalized cells, maintaining yeast fluorescence within phagocytes; and the different associations between phagocytes and fungal cells.
\end{abstract}

(c) 2014 Elsevier B.V. All rights reserved.

\section{Introduction}

Candida species are known as common colonizers of the human gastrointestinal, respiratory, and reproductive tracts. However, in immunosuppressed patients they are by far the most common cause of fungal invasive infections. Host defense against candidiasis relies mainly on the ingestion and elimination of the yeasts by the phagocytic cells (Romani, 2011). Phagocytosis of fungal pathogens is a complex process that may be divided into several distinct stages: (i) migration of phagocytes towards fungal cells; (ii) recognition of fungal pathogenassociated molecular patterns (PAMPs) through phagocyte pattern recognition receptors (PRRs); (iii) engulfment of fungal cells; and (iv) processing of engulfed cells within phagolysosomes (Lewis et al., 2012). Yeast cell wall consists of a matrix of $\beta$-glucan, chitin and mannoproteins, surrounding the plasma membrane, in which the composition and structure of cell wall mannoproteins and glucans, constitute the major yeast PAMPs (Chaffin et al., 1998). However, the cell wall is a dynamic structure and changes during cell growth and transition of yeast to hypha, changing the composition and structure of their PAMPs (Chaffin et al., 1998). Although this dynamic change is an advantage for the pathogen it hampers studies addressing the contribution of specific PAMPs to the different stages of the phagocytic process. Traditionally, studies focusing on phagocytes-yeast interaction use live yeast cells however, it was establish that the phagocytes recognize and respond to cell wall PAMPS present in both live or dead cells, with differences in the efficiency of phagocytosis only relevant after yeast

\footnotetext{
* Corresponding author at: Departamento de Biologia, Universidade do Minho, Campus de Gualtar, 4710-057 Braga, Portugal. Tel.: + 351 253601546; fax: + 351253678980.

E-mail address: psampaio@bio.uminho.pt (P. Sampaio).
}

multiplication takes place, around 45 min after co-incubation of the two types of cells (Dementhon et al., 2012).

Numerous methods have been developed to study yeast phagocytosis, being fluorescence microscopy and flow cytometry (FCM) the most reliable (Hampton and Winterbourn, 1999; Lehmann et al., 2000; Lewis et al., 2012; Linden et al., 2013). FCM combines the advantage of being fast, able to count hundreds of cells, and easily discriminate morphological heterogeneity within cell populations (Lehmann et al., 2000). In this work we evaluated the feasibility of the main phagocytosis assessing methods previously described to be adapted to FCM analysis and, to overcome some methodological limitations, we propose a new efficient method based on a combination of only two fluorochromes (Sytox Green and propidium iodide (PI)) for FCM analysis of macrophage interaction with Candida sp. cells and show that this new methodology can be used to study the contribution of different PAMPs to the phagocytic process.

\section{Materials and methods}

\subsection{Materials}

All fluorescent reagents were prepared as stock solutions and then diluted in the cell incubation medium at the desired concentrations. Fluorescein isothiocyanate (FITC) (Sigma Aldrich), was freshly prepared at $750 \mu \mathrm{g} / \mathrm{ml}$ in $0.1 \mathrm{M}$ sodium carbonate buffer ( $\mathrm{pH} 9$ ), from a $5 \mathrm{mg} / \mathrm{ml}$ dimethyl sulfoxide (DMSO) solution; Trypan Blue (TB) (Sigma Aldrich) solution (0.4\%) was used directly as stock solution; $50 \mu \mathrm{M}$ Sytox Green (Invitrogen) was prepared in PBS from a $5 \mathrm{mM}$ solution in DMSO; propidium iodide (PI) (Sigma Aldrich) was dissolved in sterile water at $1 \mathrm{mg} / \mathrm{ml}$, Calcofluor White (CFW) (Sigma Aldrich) was dissolved in 
sterile water at $10 \mathrm{mg} / \mathrm{ml}$ stock solution. Stock solutions were stored in the dark at $-20^{\circ} \mathrm{C}$

Dulbecco's Modified Eagle's medium (DMEM) was supplemented with $10 \%$ heat-inactivated fetal calf serum (FCS) (Valbiotech), $2 \mathrm{mM}$ L-glutamine, $1 \mathrm{mM}$ sodium pyruvate, and $10 \mathrm{mM}$ HEPES.

\subsection{Preparation of yeast cells}

Wild type strains of Candida albicans (SC5314), Candida glabrata (PYCC 2418), Candida bracarensis (153M), Candida parapsilosis (ATCC 22019), Candida orthopsilosis (HSM CAN 138), and Candida krusei (PYCC 3341) were used in this study. Yeast cells grown overnight at $26{ }^{\circ} \mathrm{C}$ in YEPD medium (2\% glucose, $1 \%$ bacto peptone, and $2 \%$ yeast extract) were recovered by centrifugation, washed twice in sterile $1 \times$ PBS buffer and sonicated ( 2 cycles, $5 \mathrm{~s}$ ) to eliminate aggregates.

To fix cell wall PAMPs, yeast cells were incubated for $10 \mathrm{~min}$ in formol/ethanol (1:9) and washed five times with PBS for complete removal of formol/ethanol. Yeast cells were incubated for $10 \mathrm{~min}$ with $1 \mu \mathrm{M}$ Sytox Green at room temperature (RT) in the dark, washed with PBS to remove unbound dye, and brought to the desired cell density in complete DMEM.

Live-cells were labeled with FITC or CFW. FITC, previously prepared at a concentration of $50 \mu \mathrm{g} / \mathrm{ml}$, was added directly to the yeast cells pellet and incubated for $10 \mathrm{~min}$ at room temperature in the dark. CFW was added to yeast cells at a final concentration of $5 \mu \mathrm{g} / \mathrm{ml}$ and incubated for $5 \mathrm{~min}$ at room temperature in the dark. Labeled cells were then washed with PBS to remove unbound dye, and brought to the desired cell density in complete DMEM.

\subsection{Analyses of labeled yeast cells and quenching effect}

Sytox Green, CFW or FITC labeled yeasts were incubated with TB at a final concentration of $120 \mu \mathrm{g} / \mathrm{ml}$ or with PI at a final concentration of $6 \mu \mathrm{g} / \mathrm{ml}$, in order to validate the quenching efficiency of TB or PI over Sytox Green, CFW and FITC. As neither TB nor PI enters viable cells, the internalized yeast cells will retain the original unquenched fluorescence. The $\mathrm{pH}$ fluorescence stability of the labeling dyes was evaluated by incubating labeled yeasts in a solution with $1 \mathrm{mM}$ of acetic acid $\mathrm{pH}$ of 3.9. The yeast cells were then analyzed by fluorescence microscopy or/and by flow cytometry.

\subsection{Preparation of J774.1 macrophage cell line}

The murine macrophage-like cell line J774.1 was cultured in complete DMEM at $37^{\circ} \mathrm{C}$ in a $5 \% \mathrm{CO}_{2}$ atmosphere. After confluent growth, macrophage cells were recovered, washed, and resuspended in complete DMEM to a final concentration of $5 \times 10^{5}$ cells $/ \mathrm{ml}$. For fluorescence microscopy assays, $1 \mathrm{ml}$ of the resulting cell suspension was transferred to 24-well tissue culture plates containing clean sterile glass coverslips ( $\varnothing 13 \mathrm{~mm}$ ), for flow cytometry (FMC) $3 \mathrm{ml}$ of the cell suspension were transferred to a 6 -well tissue culture plate, and for confocal microscopy, $0.2 \mathrm{ml}$ of the cells were plated in a microscopy chamber plate (Ibidi). Cells were then incubated overnight, at $37^{\circ} \mathrm{C}$ in a $5 \% \mathrm{CO}_{2}$ atmosphere, to allow macrophage adherence. In the following day, phagocytic cells were washed twice with $1 \times$ PBS buffer to remove non-adherent cells, and cells were promptly used for phagocytic assays. Triplicates were done in each plate.

\subsection{Phagocytosis of Candida cells}

Macrophages were incubated with labeled yeast suspensions at MOI of $1 \mathrm{M}: 5 \mathrm{Y}$ for $30 \mathrm{~min}$, at $37^{\circ} \mathrm{C}$ and $5 \% \mathrm{CO}_{2}$. After incubation plates were kept on ice to stop phagocytosis, and wells rinsed twice with PBS to remove unbound yeasts. Macrophages and associated yeasts were then incubated with TB at a final concentration of $120 \mu \mathrm{g} / \mathrm{ml}$ or with PI at a final concentration of $6 \mu \mathrm{g} / \mathrm{ml}$, for $5 \mathrm{~min}$ at RT. Cells were analyzed by fluorescence microscopy (Leica DM5000B), confocal microscopy (Leica SP2 AOBS SE) and by flow cytometry (EPICS XL-MCL, Beckman-Coulter Corporation). Fluorescence microscopy images were analyzed using ImageJ cell counter software. Confocal microscopy images were analyzed using Fiji software. FCM data was analyzed by using Flowing software (version 2.5; Turku Centre for Biotechnology, University of Turku), as indicated in the following section.

The statistical significance values were tested by means of repeated ANOVA measures. Multiple comparisons were performed according to the Bonferroni test using GraphPad Prism 5 software (GraphPad Software, Inc., La Jolla, CA).

\subsection{Flow cytometry (FCM) analysis}

FCM analysis was performed on an EPICS XL-MCL (Beckman-Coulter Corporation, Hialeah, FL, USA) flow cytometer. At least twenty thousand cells were analyzed per sample at low flow rate. An acquisition protocol was defined to measure forward scatter (FSC), side scatter (SSC), green fluorescence and red fluorescence on a logarithmic scale. Each sample was collected for $30 \mathrm{~s}$ at the slowest flow rate to minimize the coincidental appearance of free yeasts and macrophages in the laser beam. A FSC threshold was set to gate out debris. Macrophages and free yeast cells were discriminated by combined measurements of FSC and SSC. Macrophages were gated in R1 area and free yeast cells in R2 area. The control with macrophages alone was used to define the R1 gates.

The percentage of yeast particles interacting with macrophages was calculated from dot plot analysis of Sytox Green fluorescence intensity vs. PI fluorescence intensity of R1-gated events by using Flowing software. The percentage of macrophages with attached yeast particles $\left(\mathrm{M}_{\mathrm{YA}}\right)$ was calculated using the next formula, following the red fluorescence signal:

$\mathrm{M}_{\mathrm{YA}}=\left(\mathrm{M}_{\mathrm{R}} * 100 / \mathrm{M}_{\mathrm{AF}}\right)$,

where $M_{R}$ is the mean red fluorescence (calculated by number of events in quadrant $\mathrm{Q} 4$ ), gated in $\mathrm{R} 1, \mathrm{M}_{\mathrm{AF}}$ is the total no. of events gated in R1. The percentage of macrophages with ingested and attached yeast particles ( $\mathrm{M}_{\mathrm{YAI}}$ ) was assessed as follows:

$\mathrm{M}_{\mathrm{YAI}}=\mathrm{M}_{\mathrm{RG}} * 100 / \mathrm{M}_{\mathrm{AF}}$,

where $M_{R G}$ is the mean green and red fluorescence (calculated by number of events in quadrant Q3), gated in R1. The percentage of macrophages with only ingested yeast particles $\left(\mathrm{M}_{\mathrm{YI}}\right)$ was assessed as follows:

$\mathrm{M}_{\mathrm{YI}}=\mathrm{M}_{\mathrm{G}} * 100 / \mathrm{M}_{\mathrm{AF}}$,

where $M_{G}$ is the mean green fluorescence (calculated by number of events in quadrant Q2) gated in R1. The percentage of phagocytosis, macrophages with ingested yeast particles $\left(\mathrm{M}_{\mathrm{YI}}\right)$, was assessed as follows:

$\mathrm{M}_{\mathrm{YF}}=\mathrm{M}_{\mathrm{YI}}+\mathrm{M}_{\mathrm{YA}}$

\section{Results and discussion}

\subsection{Analyses of labeled yeast cells and quenching effect}

The challenge in developing an in vitro method to assess phagocytosis of yeast cells based on flow cytometry (FCM) relies on the difficulty to distinguish between internalized and non-internalized yeasts, and on the stability of the fluorochrome within the hostile environment of the phagolysosome both reducing the accuracy of phagocytosis measurements (Lehmann et al., 2000). In this view, these two characteristics were analyzed with several fluorochrome combinations in order to assess their utility for phagocytosis measurements by FCM. 
One way to distinguish between internalized and non-internalized yeasts is by taking advantage of the quenching effect of some dyes over the emitted fluorescence of another dye. So, first we tested the ability of TB or PI in quenching FITC fluorescence on labeled yeast cells. We observed that TB, although not completely, was able to quench FITC fluorescence, as described in previous studies (Busetto et al., 2004; Warolin et al., 2005) but FITC fluorescence is no longer detected upon incubation at a lower pH, as previously reported (Hoch et al., 2005). PI was not able to quench FITC fluorescence (data not shown). Thus, both combinations FITC/PI and FITC/TB were discarded for phagocytosis measurements by FCM. The quenching effect of TB over CFW-stained yeast cells was also tested and results confirmed previous data that TB quenches CFW fluorescence (Dementhon et al., 2012) but, CFW fluorescence is significantly reduced at lower $\mathrm{pH}$ (data not shown). Once again, PI failed as a quenching dye, being unable to quench CFW fluorescence (data not shown).

Traditionally, studies focusing on phagocytes-yeast interaction use live yeast cells, however, it was already establish that the innate immune cells recognize and respond to cell wall PAMPs in both, live or dead cells. The major difference described is in the efficiency of phagocytosis but this difference is only relevant after yeast multiplication takes place (Dementhon et al., 2012). However, inactivating treatments based on heat killing by boiling result in the release of matricial cell wall surface components (Sarazin et al., 2010), which are extremely important for phagocyte recognition. On the contrary, inactivating treatments based on fixation of the cell wall components conserve the PAMPs in such a way that enables antibody recognition (Murciano et al., 2007). In this sense, in the present study yeast cells were inactivated by a fixation method that does not alter the fungal cell wall structure.
The use of dead yeast cells allowed the exploitation of viability fluorescent dyes such as Sytox Green for yeast labeling, avoiding the surface exposure of the dyes. Sytox Green is a high affinity nuclear stain that penetrates cells and intercalates with nucleic acids, staining the cell. The ability of TB or PI to quench the Sytox Green-stained yeast cells was also assessed (Fig. 1). As can be observed in the figure, TB incompletely quenches Sytox Green fluorescence however, the quenching effect over Sytox Green labeled yeasts is remarkably evident when PI is used. Additionally, no significant reduction of fluorescence was observed when Sytox Green labeled yeast cells were incubated at pH 3.9 (Fig. 1).

Since Sytox Green/PI combination is more adequate for flow cytometry analyses than Sytox Green/TB, the quenching effect and pH stability of the Sytox Green/PI protocol were confirmed by FCM. Non-stained yeast cells were gated (Fig. 2A) and used to calibrate yeast autofluorescence in the cytometer (Fig. 2B). After incubation with Sytox Green, 99.97\% of the yeast cells are labeled, validating Sytox Green label efficiency (Fig. 2C). The incubation of Sytox Green labeled cells with PI shifted the fluorescence of $99.25 \%$ of the cells to PI positive, while only $0.75 \%$ remained Sytox Green positive (Fig. 2D), confirming our previous results of an almost complete quenching of the Sytox Green fluorescence. The mean fluorescence (MFI) of Sytox Green yeast cells incubated at pH 3.9 showed a slight decrease in intensity but yeast cells remained Sytox Green stained, supporting our previous observations (Fig. 3 ).

In order to evaluate the efficiency of Sytox Green/PI protocol in a phagocytosis assay, fixed C. albicans cells stained with Sytox Green were incubated with macrophages for $30 \mathrm{~min}$ and then stained with PI. The murine macrophage-like cell line J774.1 was selected for this study, since it is widely used in phagocytic studies and no significant

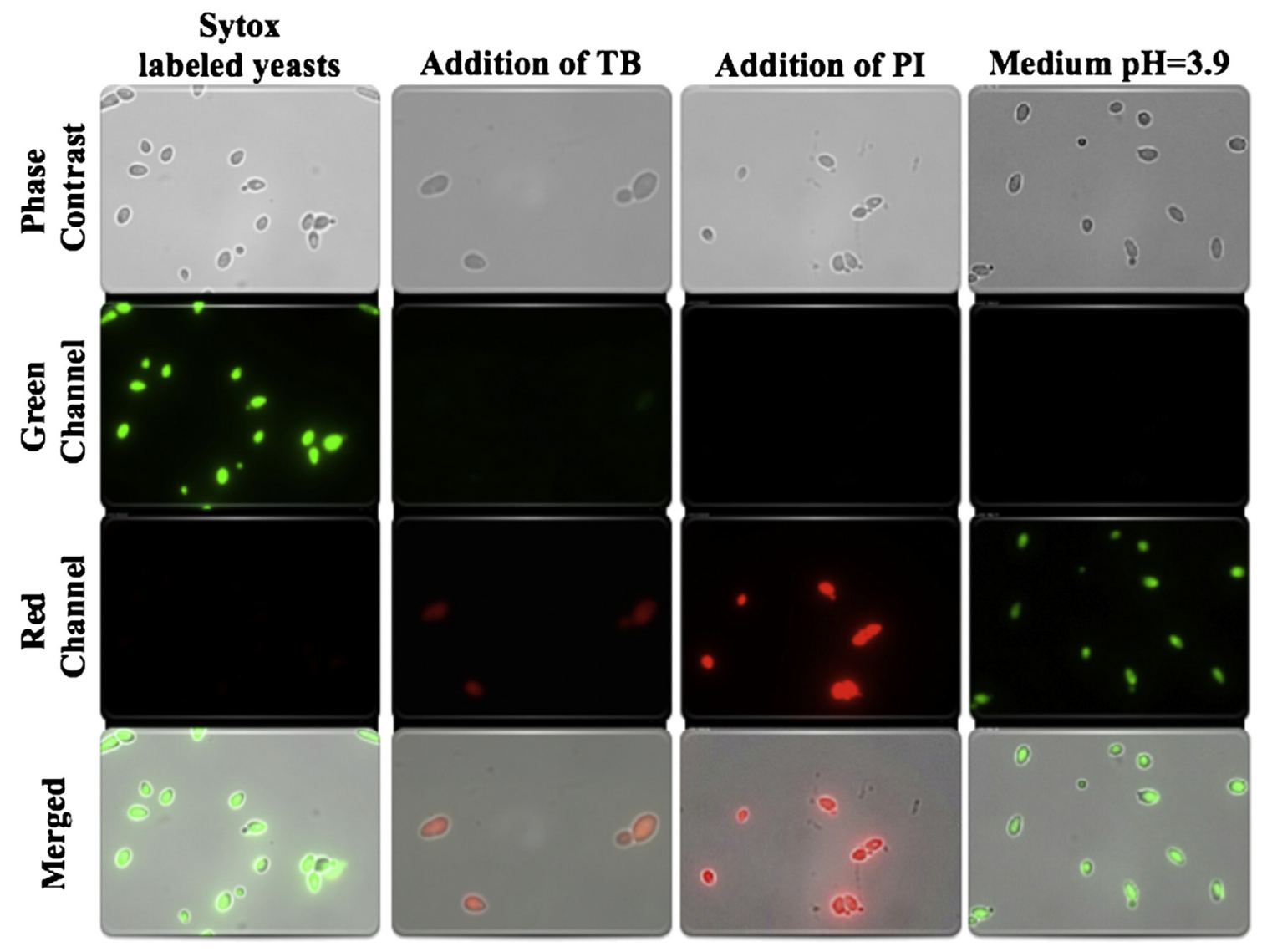

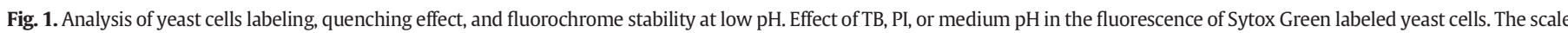
bar represents $7.5 \mu \mathrm{m}$. 

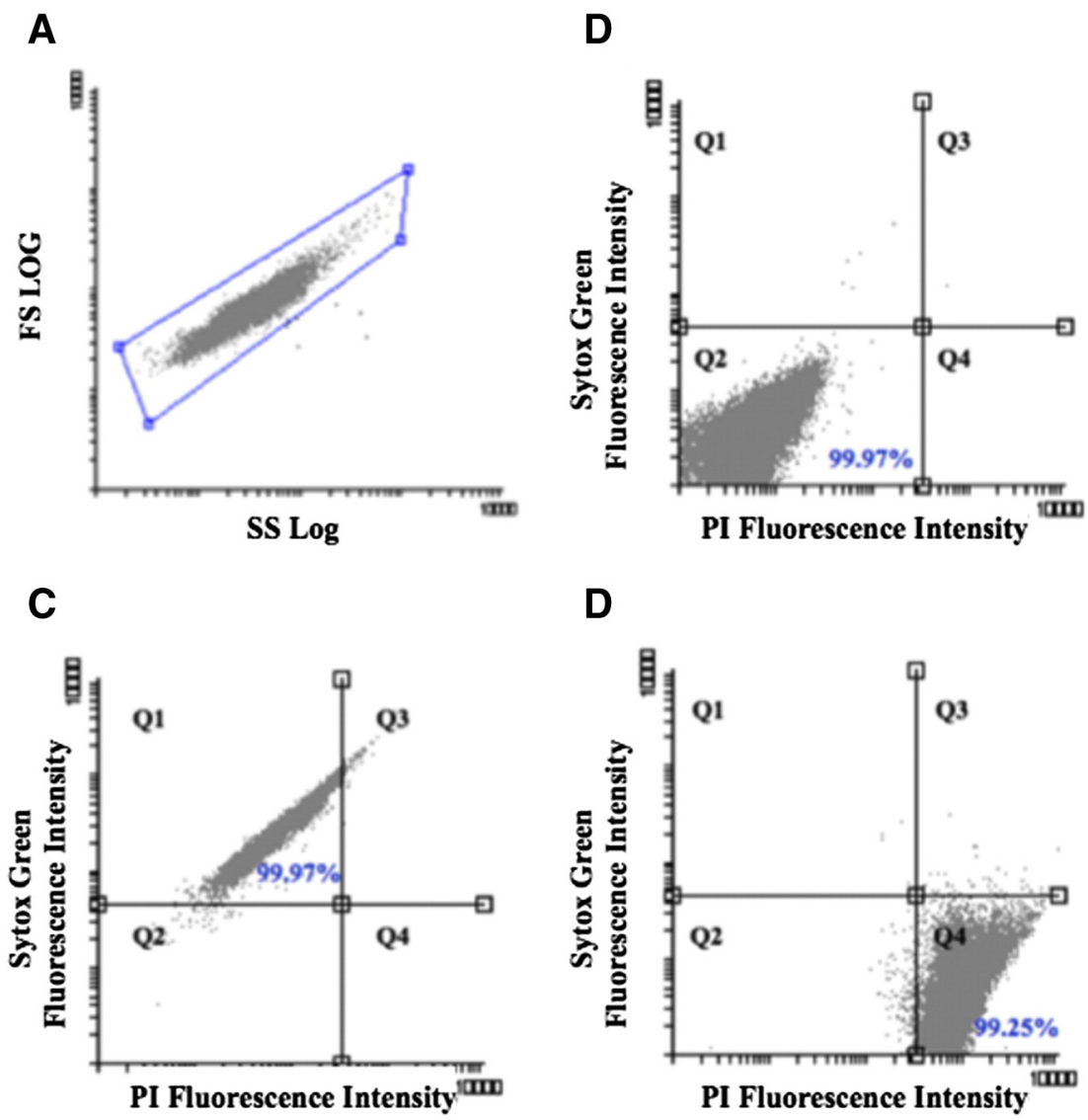

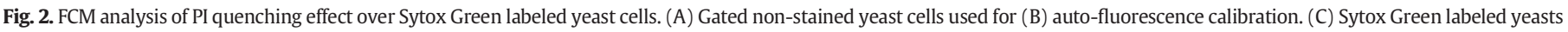
showing green fluorescence (Q1) and (D) Sytox Green/PI double-stained yeast cells showing red florescence (Q4).

differences regarding the ability to engulf yeast cells are described, in comparison with primary phagocytic culture, at least within the time point used in our study (Lewis et al., 2012). The confocal microscopy analysis after phagocytosis revealed, as expected, that without fluorescence distinction of internalized from non-internalized yeast cells is imprecise (Fig. 4Ai). The PI quenching effect of the non-internalized yeast cells (Fig. 4Aiii), and the integrity of the Sytox Green fluorescence of C. albicans cells inside live macrophages (Fig. 4Aii) are clearly visible

1)

2) 3)

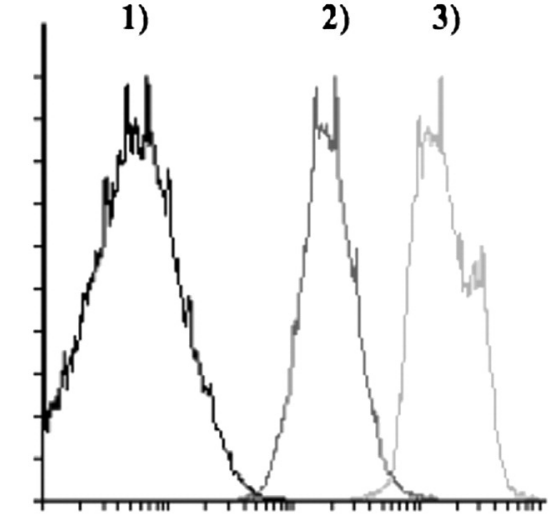

Sytox Green Fluorescence Intensity

1) Non-stained yeasts

2) Sytox Green labelled yeasts medium $\mathrm{pH}=3.9$

3) Sytox Green labelled yeasts medium $\mathrm{pH}=7.5$

Fig. 3. FCM analysis of fluorescence stability at low pH in Sytox Green labeled yeast cells. Histogram overlay of Sytox Green Medium Fluorescence Intensity (MFI) of non-labeled yeast cells (1), labeled yeast cells at pH of 7.5 (2), and labeled yeast cells at pH of 3.9 (3). and critical for the accurate distinction. In order to confirm that Sytox Green labeled yeast cells were indeed inside macrophages, an ortho stack 3D visualization of the cells is represented in Fig. 4B (Movie S1). In view of these results, the presented protocol will enable differentiation of: (i) non labeled phagocytes, representing phagocytes with no interaction with yeast cells; (ii) Sytox Green labeled phagocytes, representing phagocytes that have only internalized yeast cells; (iii) PI labeled phagocytes, representing phagocytes that have only attached yeast cells; and (iv) Sytox Green and PI labeled phagocytes, representing phagocytes that have both internalized and attached yeast cells.

\subsection{FCM analysis of the phagocytosis assay with different Candida species}

In order to test this new FCM phagocytosis protocol, assays were performed using different Candida species: C. albicans, C. glabrata, C. bracarensis, C. parapsilosis, C. orthopsilosis, and C. krusei. The \% of phagocytes with internalized yeast cells, the \% of phagocytes that have both internalized and attached yeast cells, and the \% of phagocytes that have only attached yeast cells were calculated. Before incubation with yeast cells macrophage auto-fluorescence was calibrated with a suspension of non-stained macrophages. Then, PI stained macrophages were evaluated and two populations clearly distinguishable, one representing dead macrophages (PI positive), which was not analyzed, and the other, the live macrophages, designated as R1 (P1 negative) (Fig. 5A). The non stained R1 macrophages were indeed PI negative and were further analyzed in a second dot plot, by plotting Sytox Green fluorescence against PI fluorescence. The majority of the cells (97.39\%) gated in a quadrant (Q2) were used to define the limits of Sytox Green and PI negative cells (Fig. 5B). The other quadrants will gate the distinct phagocytes-yeast interactions: phagocytes that have only internalized yeast cells (Q1), phagocytes representing phagocytes 
A

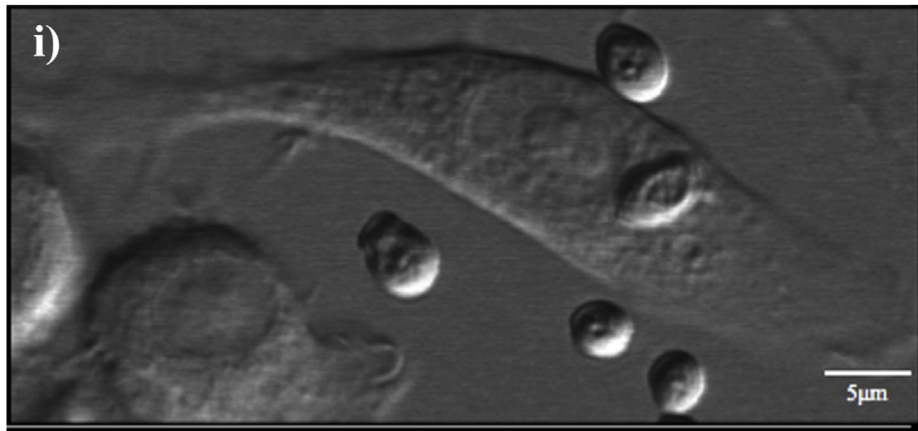

ii)

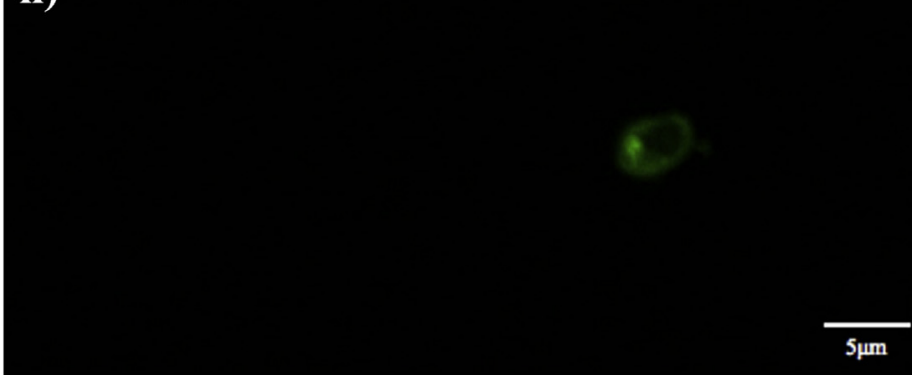

$5 \mu \mathrm{m}$

iii)

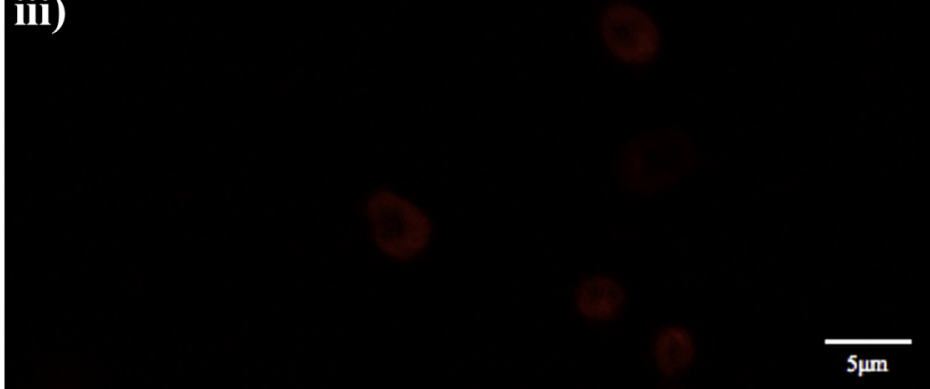

iv)

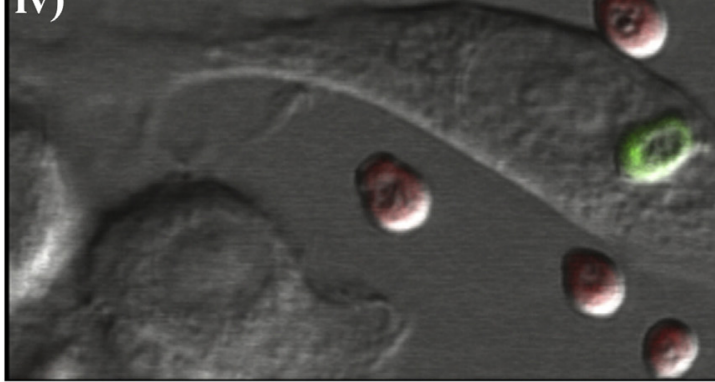

B
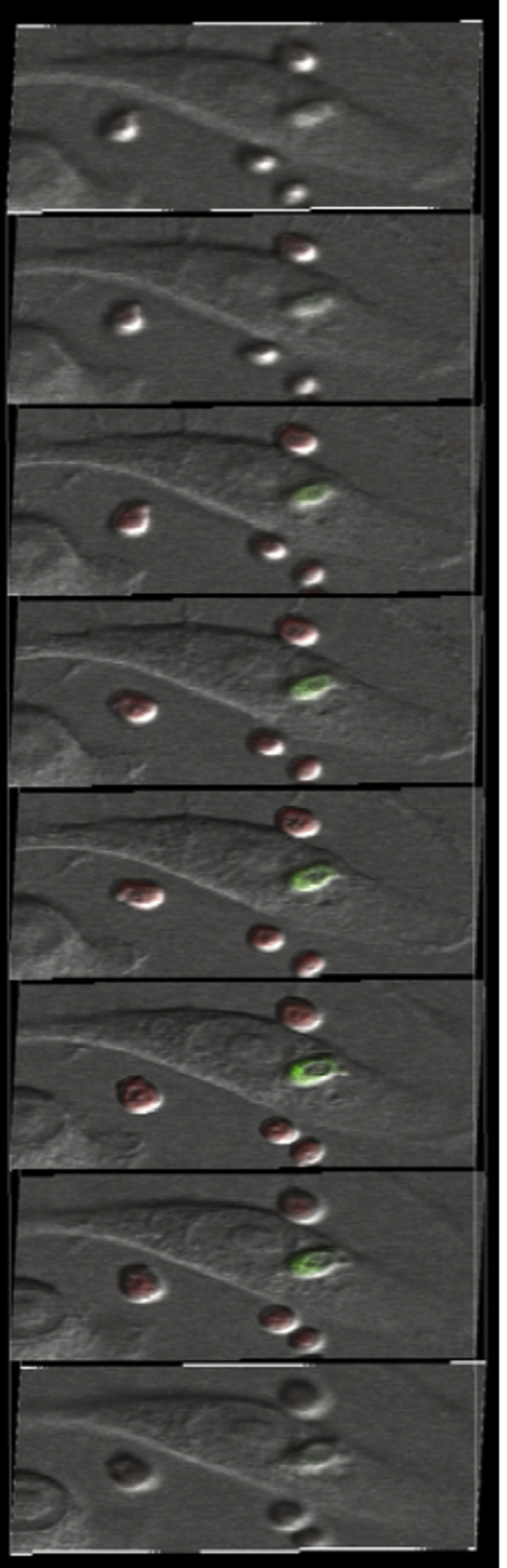

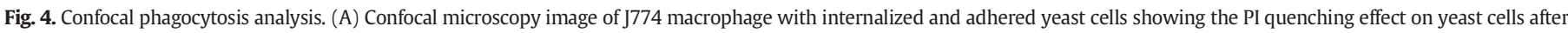

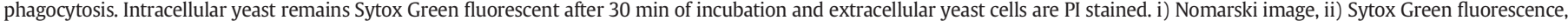
iii) PI fluorescence and iv) merged image. (B) Z-stack images were analyzed to confirm internalization. The scale bar represents $5 \mu \mathrm{m}$.

that have both internalized and attached yeast cells (Q3), and phagocytes that have only attached yeast cells (Q4).

In a first approach, $C$. albicans FCM assessed phagocytosis was performed to test the protocol. Dot plot of macrophages after incubation with the yeast cells showed an additional population, the free yeast cells (R2), that were not analyzed (Fig. 5C). In order to quantify C. albicans phagocytosis, the R1 cell population was selected and the fluorescence intensity of Sytox Green vs. PI analyzed, by using the previously defined gate limits (Fig. 5D). Four distinct phagocytes-yeast interactions could be quantified: in Q1 the phagocytes that have only internalized yeast cells $\left(\mathrm{M}_{\mathrm{YI}}, 57.6 \%\right)$; in Q2 the phagocytes with no interaction with yeast cells ( $\left.\mathrm{M}_{\mathrm{YNO}}, 42.7 \%\right)$; in Q3 the phagocytes with both internalized and attached yeast cells ( $\left.\mathrm{M}_{\mathrm{YAl}}, 8.7 \%\right)$; and in $\mathrm{Q} 4$ the phagocytes with only attached yeast cells ( $\left.\mathrm{M}_{\mathrm{YA}}, 1.50 \%\right)$. These results confirmed that the new fluorochrome combination is suitable for flow cytometry phagocytosis assays of fixed yeast cells. Following this confirmation, the protocol was extended to evaluate phagocytosis quantified by the new protocol in different Candida species. As expected, different species presented different ratios of macrophage ingestion and/or adhered yeast cells (Fig. 6). C. orthopsilosis was the species with the higher percentage of ingested/adhered cells followed by C. krusei, C. bracarensis and C. parapsilosis, while C. glabrata and C. albicans were the species with the lower percentage. These results are in accordance with several studies that demonstrated that the immune cells respond differently 
A
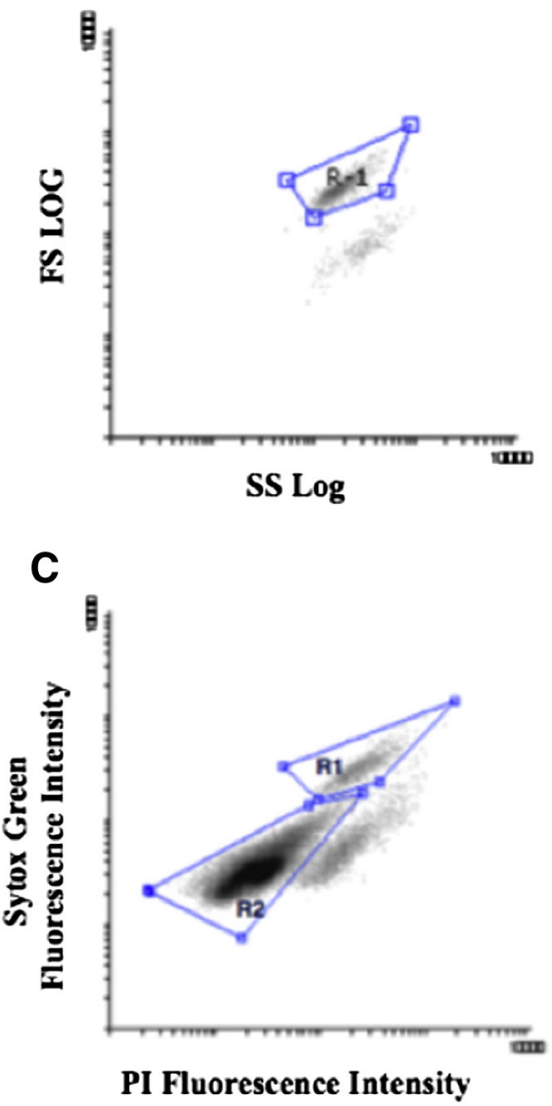

B

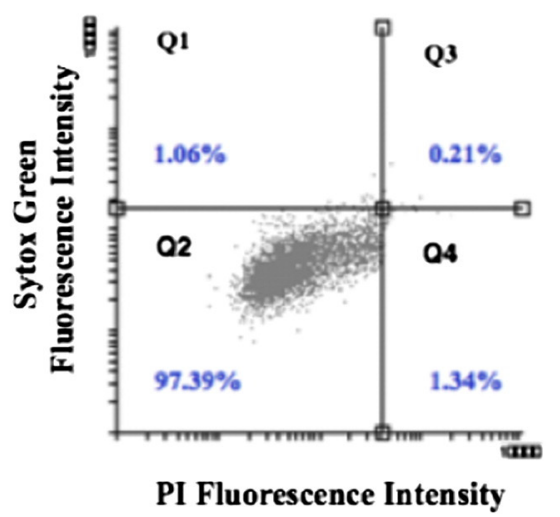

D

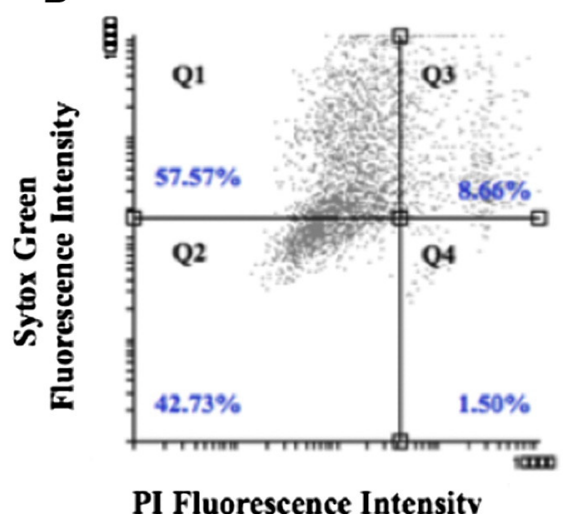

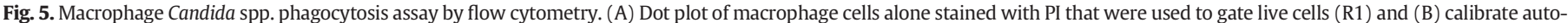

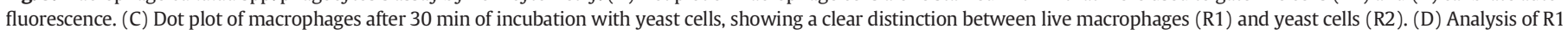

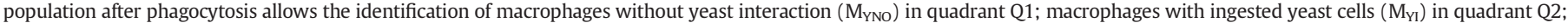
macrophages with ingested and adhered yeast cells ( $\mathrm{M}_{\mathrm{YAI}}$ ) in quadrant $\mathrm{Q} 3$, and macrophages with only adhered cells ( $\mathrm{M}_{\mathrm{YA}}$ ) in quadrant $\mathrm{Q} 4$.

to individual Candida species in part, due to differences in cell wall PAMP components and organization (de Groot et al., 2008; Keppler-Ross et al., 2010; Kuhn and Vyas, 2012; McKenzie et al., 2010; Mora-Montes et al., 2012; Sheth et al., 2011). It was also described that phagocytosis was more efficient using $C$. parapsilosis as a target than C. albicans yeasts (Destin et al., 2009), C. krusei was more phagocytized than C. albicans or C. glabrata (Wellington et al., 2003), and C. orthopsilosis was less resistant to phagocytic host defenses than C. parapsilosis (Sabino et al., 2011).

For methodology comparison, fluorescence microscopy images were taken and analyzed to assess the percentage of macrophages with internalized C. albicans, C. parapsilosis and C. orthopsilosis yeast cells. At least 500 macrophages were counted in each slide and results confirmed that C. orthopsilosis is easily internalized followed by C. parapsilosis and by C. albicans (Fig. S1). The percentages of internalization were slightly lower than those observed with FCM, probably due to the fact that the number of macrophages counted was 10 times lower than those assessed by flow cytometry.

\section{Conclusion}

In conclusion, we proposed a new, fast and simple method to study the interaction between phagocytes and Candida yeast cells and to quantify phagocytosis. The method is based on the quenching effect of PI over Sytox Green fluorescence and allows a clear distinction between internalized and non-internalized yeast cells with the advantage of maintaining Sytox Green fluorescence upon internalization of the yeast by phagocytic cells. Using only these two fluorochromes we could accurately distinguish and quantify various parameters simultaneously, in a single infection experiment: i) phagocyte survival and ii) the association of phagocytes to fungal cells: phagocytes that have only internalized yeast cells, phagocytes that have only attached yeast cells, and phagocytes that have both, internalized and attached yeast cells. In addition, this method proved to be efficient for evaluation of differences in the phagocytosis of distinct Candida species. Although the comparative virulence among different Candida species is not entirely dependent on its recognition and internalization by phagocytes, the innate immune system plays an important role in controlling Candida infections, and this technique may help to elucidate some PAMPs characteristics that may be involved in the virulence process. Contrary to microscopy, which may be too cumbersome for analyzing a large number of strains, this method allows the high-throughput screening of yeast strains from different species, including mutants. Such studies would significantly enhance our understanding of the molecular mechanisms involved in the interaction between yeasts and host phagocytes.

Supplementary data to this article can be found online at http://dx. doi.org/10.1016/j.mimet.2014.03.013.

\section{Acknowledgments}

We thank Professor Paula Sampaio from IBMC (Institute for Molecular and Cell Biology) for helping with the confocal microscopy. 


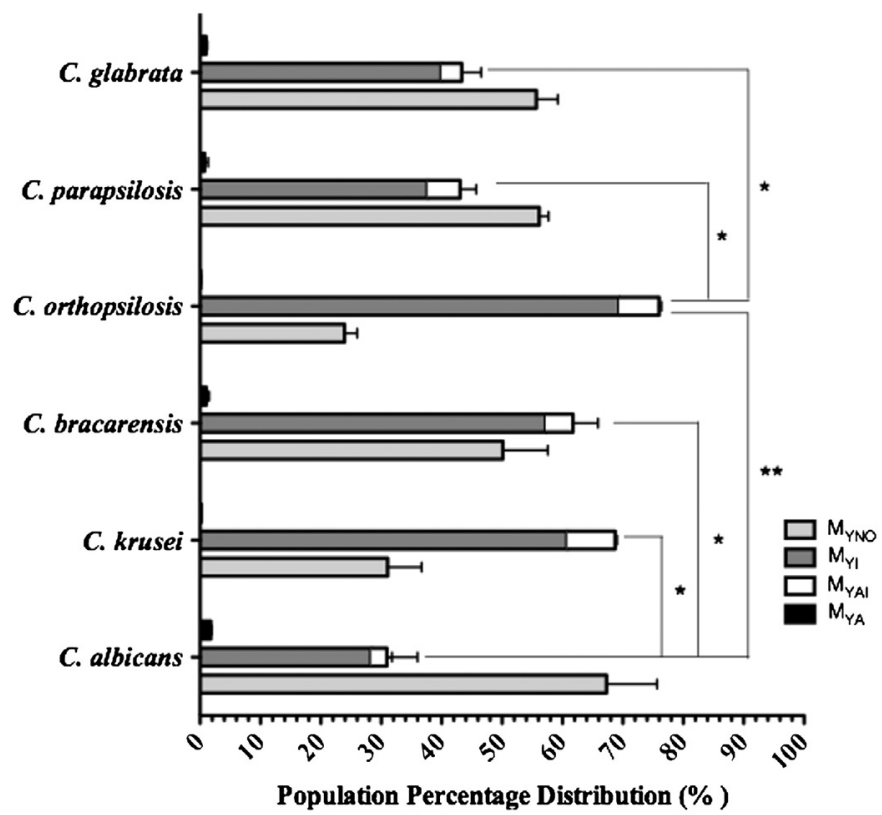

Fig. 6. Phagocytosis assay with cells from different Candida species. Analysis of the interactions of $\mathrm{J774}$ macrophages with formol/ethanol (1:9) killed Candida sp. cells after $30 \mathrm{~min}$ of incubation. The light gray bars $\left(\mathrm{M}_{\mathrm{YNO}}\right)$ represent the \% of macrophages with no interaction with yeast cells; The gray bars $\left(\mathrm{M}_{\mathrm{YI}}\right)$ macrophages with internalized yeast cells; white bars (M MAI $)$ macrophages with internalized and adhered yeast cells and the black bars $\left(M_{Y A}\right)$ macrophages with only adhered cells. The gray bar together with the white bar represents the \% of phagocytosis. Each bar is the average of two independent experiments. The significance of the results between the different species is indicated in this figure $\left({ }^{*} \mathrm{P}<0.05,{ }^{* *} \mathrm{P}<0.01\right)$.

This research was supported by FEDER Funds through the Operational Programme COMPETE and national funds through the Fundação para a Ciência e Tecnologia (FCT), in the scope of project PEst-OE/BIA/ UI4050/2014.

\section{References}

Busetto, S., et al., 2004. A single-step, sensitive flow cytofluorometric assay for the simultaneous assessment of membrane-bound and ingested Candida albicans in phagocytosing neutrophils. Cytometry. A 58 (2), 201-206.
Chaffin, W.L., et al., 1998. Cell wall and secreted proteins of Candida albicans: identification, function, and expression. Microbiol. Mol. Biol. Rev. 62 (1), 130-180.

de Groot, P.W., et al., 2008. The cell wall of the human pathogen Candida glabrata: differential incorporation of novel adhesin-like wall proteins. Eukaryot. Cell 7 (11), 1951-1964.

Dementhon, K., El-Kirat-Chatel, S., Noel, T., 2012. Development of an in vitro model for the multi-parametric quantification of the cellular interactions between Candida yeasts and phagocytes. PLoS One 7 (3), e32621.

Destin, K.G., et al., 2009. Oxidative burst and phagocytosis of neonatal neutrophils confronting Candida albicans and Candida parapsilosis. Early Hum. Dev. 85 (8), 531-535.

Hampton, M.B., Winterbourn, C.C., 1999. Methods for quantifying phagocytosis and bacterial killing by human neutrophils. J. Immunol. Methods 232 (1-2), 15-22.

Hoch, H.C., et al., 2005. Two new fluorescent dyes applicable for visualization of fungal cell walls. Mycologia 97 (3), 580-588.

Keppler-Ross, S., et al., 2010. Recognition of yeast by murine macrophages requires mannan but not glucan. Eukaryot. Cell 9 (11), 1776-1787.

Kuhn, D.M., Vyas, V.K., 2012. The Candida glabrata adhesin Epa1p causes adhesion, phagocytosis, and cytokine secretion by innate immune cells. FEMS Yeast Res. 12 (4), 398-414.

Lehmann, A.K., Sornes, S., Halstensen, A., 2000. Phagocytosis: measurement by flow cytometry. J. Immunol. Methods 243 (1-2), 229-242.

Lewis, L.E., et al., 2012. Stage specific assessment of Candida albicans phagocytosis by macrophages identifies cell wall composition and morphogenesis as key determinants. PLoS Pathog. 8 (3), e1002578.

Linden, J.R., et al., 2013. The role of galectin-3 in phagocytosis of Candida albicans and Candida parapsilosis by human neutrophils. Cell. Microbiol. 15 (7), 1127-1142.

McKenzie, C.G., et al., 2010. Contribution of Candida albicans cell wall components to recognition by and escape from murine macrophages. Infect. Immun. 78 (4), 1650-1658.

Mora-Montes, H.M., et al., 2012. Interactions between macrophages and cell wall oligosaccharides of Candida albicans. Methods Mol. Biol. 845, 247-260.

Murciano, C., et al., 2007. Both viable and killed Candida albicans cells induce in vitro production of TNF-alpha and IFN-gamma in murine cells through a TLR2-dependent signalling. Eur. Cytokine Netw. 18 (1), 38-43.

Romani, L., 2011. Immunity to fungal infections. Nat. Rev. Immunol. 11 (4), 275-288.

Sabino, R., et al., 2011. Isolates from hospital environments are the most virulent of the Candida parapsilosis complex. BMC Microbiol. 11, 180.

Sarazin, A., Poulain, D., Jouault, T., 2010. In vitro pro- and anti-inflammatory responses to viable Candida albicans yeasts by a murine macrophage cell line. Med. Mycol. 48 (7), 912-921.

Sheth, C.C., et al., 2011. Glycosylation status of the C. albicans cell wall affects the efficiency of neutrophil phagocytosis and killing but not cytokine signaling. Med. Mycol. 49 (5), 513-524.

Warolin, J., Essmann, M., Larsen, B., 2005. Flow cytometry of Candida albicans for investigations of surface marker expression and phagocytosis. Ann. Clin. Lab. Sci. 35 (3), 302-311.

Wellington, M., Bliss, J.M., Haidaris, C.G., 2003. Enhanced phagocytosis of Candida species mediated by opsonization with a recombinant human antibody single-chain variable fragment. Infect. Immun. 71 (12), 7228-7231. 